\title{
Toward specific ways to combine ketamine and psychotherapy in treating depression
}

\author{
Gregor Hasler* ${ }^{*}$
}

Unit of Psychiatry Research, University of Fribourg, Chemin du Cardinal-Journet 3, Fribourg, Switzerland

In major depression, remission rate in response to monoaminergic antidepressant is around $50 \%$. The lack of strong synergies between classical antidepressants and psychotherapy may be due to the molecular effects of classical antidepressants. They modulate synapses but they do not substantially influence synaptogenesis. They also increase brain-derived neurotrophic factor (BDNF). However, for activity-dependent plasticity, BDNF release has to work in concert with activation of synaptogenesis. There has been considerable excitement about ketamine's antidepressant effect. Ketamine leads to fast changes in synaptic function and plasticity that go well beyond effects of classical antidepressants. As a result, ketamine may turn out to have the capacity to considerably enhance the effects of psychotherapy. Such enhancing effects may become an important clinical indication for ketamine since its purely pharmacological effect is transient. This editorial outlines some mechanistic hypotheses, how Behavioral Activation, Trauma-Focused Psychotherapies and Humanistic Psychotherapy may specifically prolong ketamine's antidepressant effects.

Received 4 April 2019; Accepted 9 April 2019; First published online 19 June 2019

Key words: depression, psychology, pharmacology, neuroplasticity.

Major depressive disorder (MDD) and depressive episodes in the course of bipolar disorders (BD) are a major public health concern. In MDD, the remission rate in response to classical, monoaminergic antidepressants is $40-60 \%$ in clinical trials, and $20-40 \%$ in naturalistic settings. Numbers are even lower for bipolar depression. The persistence of depressive symptoms in MDD and BD have been associated with increased risk of relapse, social, and occupational impairments, cognitive deficits, and increased suicide risk.

The benefits of combining psychotherapy with classical antidepressants are a matter of debate. Meta-analyses have consistently found small benefits from combination treatment over treatment with an antidepressant or psychotherapy alone. However, it remains unclear whether these benefits are clinically relevant. ${ }^{1,2}$

All types of psychotherapies involve learning and behavioral change. One likely source of synergies between pharmacotherapy and psychotherapy is the neuroplasticity-enhancing effect of psychotropics that enables lasting cognitive restructuring and behavioral change. The lack of strong synergies between classical antidepressants and psychotherapy in MDD may be due

*Address correspondence to: Gregor Hasler (http://orcid.org/00000002-8311-0138), Psychiatry Research Unit, University of Fribourg, Chemin du Cardinal-Journet 3, 1752 Villars-sur-Glâne, Switzerland. (Email: gregor.hasler@unifr.ch) to the molecular effects of classical antidepressant drugs on neuroplasticity. These drugs modulate synapses, but they do not substantially influence synaptogenesis. They also increase brain-derived neurotrophic factor (BDNF). However, for activity-dependent formation and plasticity, BDNF release has to work in concert with activation of synaptogenesis. ${ }^{3}$

There has been considerable excitement about the discovery of ketamine's antidepressant effect when given at a sub-anesthetic dose. Ketamine has a clearly different therapeutic mechanism than classical antidepressants, which is of clinical importance. For example, the onset of action is more rapid, it effectively reduces suicidal risk, and it is effective in patients who frequently do not respond to monoaminergic antidepressants, for example, patients with bipolar depression and depressed patients who also suffer from obesity.

Ketamine is an antagonist at the N-methyl-D-aspartate (NMDA) receptor. However, the therapeutic mechanisms are way more complex than this receptor blockade. Particularly, ketamine leads to fast changes in synaptic function and plasticity that go well beyond effects on neuroplasticity of classical antidepressant compounds. Ketamine increases mTORC1 signaling, synaptic number, and function. These changes persist over a week and even longer and correlate with the mitigation of depressive symptoms. $^{3}$ 
As a result, ketamine may turn out to have the capacity to considerably enhance the effects of psychotherapy in treatment-resistant unipolar and bipolar depression. In fact, such an enhancing effect has the potential to become an important clinical indication for ketamine. The transient nature of ketamine's antidepressant effects and the potentially severe side effects of a long-term treatment with repeated applications has raised concerns about the use of this new antidepressant in clinical settings. As a result, the identification of strategies to enhance the sustainability of ketamine's initial strong antidepressant effect has become an important research question in the field. ${ }^{4}$

There is preliminary evidence that cognitive behavioral therapy (CBT) extends the duration of ketamine's antidepressant effects. ${ }^{5}$

Ketamine alters activity in brain circuits related to reward and motivation. ${ }^{5}$ In a clinical study on MDD and $\mathrm{BD}$, patients with at least moderate anhedonia at baseline were found to be $55 \%$ more likely to respond to IV ketamine. ${ }^{6}$ Moreover, ketamine's pronounced pro-hedonic effects have been associated with ketamine's antisuicidal effects. $^{7}$ These findings are interesting in the context of behavioral models of depression that emphasize low positive reinforcement as a causal factor of depressed states.

The main goal of behavioral activation, a highly effective form of psychotherapy for depression, is to increase environmental reinforcement. Subjective experience of environmental reward, rather than objective reward, mediates the relationship between avoidance and mitigation of depression. As a consequence, this approach works only if the human reward pathways are responsive and plastic. Therefore, in MDD and BP with pronounced anhedonia, the combination of ketamine and behavioral activation appears to be a highly promising approach. The behavioral change resulting from such a combination therapy may last much longer than ketamine's transient antidepressant effects.

Several studies have demonstrated that inflammation is a predictor of poor response to CBT. ${ }^{8}$ Unspecific effects such as cognitive deficits related to memory, attention, learning, and fatigue may underlie this relationship. In addition, inflammation has specific effects on social cognition and behaviors, such as feelings of social disconnection, impaired social cognitive processing, and increased rejection sensitivity. These symptoms may have had an evolutionary survival function. However, in modern times, they can reduce the effectiveness of psychosocial interventions, particularly of interpersonal psychotherapies. Thus, ketamine has the potential to enhance the effects of interpersonal and psychodynamic therapies by its anti-inflammatory effects.

Attentional and mnemonic biases toward negative information and emotions are one of the most consistent neuropsychological findings in MDD that may even play a causal role in the development of depressive disorders. ${ }^{9}$ In our own, we found in unpublished data that ketamine reduced pessimism, which is consistent with the finding that ketamine blocks lateral habenula bursting. ${ }^{10}$ The combination of ketamine with cognitive bias modification therapy may prolong ketamine's positive neuropsychological effect.

Unfortunately, we do not yet know the full amount of possible diverse effects of low-dose ketamine, as administered in MDD and BP, on cognition. However, there is consistent preclinical evidence that ketamine has a complex effect on memory, including disruption of fear memory consolidation and strengthening the formation of extinction memory. ${ }^{11}$ These data suggest that ketamine may have the potential to enhance exposure-based therapies in traumatized depressed patients and those with phobias.

Regarding neuroplastic effects, there may be important similarities between ketamine and other putative antidepressants that increase glutamate release, including serotonergic hallucinogens such as LSD and psilocybin. ${ }^{5}$ At the clinical level, serotonergic hallucinogens have the potential to induce long-term changes in certain personality traits. In particular, there is preliminary evidence that psilocybin increased the Big Five personality trait Openness. This therapeutic change lasted over 1 year. ${ }^{12}$ Carl Rogers, the highly influential founder of humanistic psychotherapy, considered openness as a core feature of therapeutic change and the fully functionally patient: "He is able to experience all of his feelings, and is afraid of none of his feelings. He is his own sifter of evidence, but he is open to evidence from all sources ... because of the awareness of himself which flows freely in and through his experience, he is a fully functioning person."13 If ketamine has similar effects on openness as hallucinogens, it may have the potential to become an important enhancer of humanistic psychotherapies that are popular among therapists and patients in naturalistic settings.

Recently, we demonstrated that ketamine can change body feelings and induce subjective lightness and floating, ${ }^{14}$ which suggests a link between ketamine, body therapy, and mindfulness-based psychotherapies.

Depression is frequently associated with social anhedonia, increased rejection sensitivity, impaired social communication, and lack of empathy. ${ }^{15}$ In our clinical experience, ketamine rapidly improved social functioning in depressed patients. As a consequence, it facilitated to build up a therapeutic relationship, which is the basis of almost all psychotherapies.

In sum, the transient nature of ketamine's antidepressant effects in concert with growing evidence on its neuroplasticity-related therapeutic mechanisms encourages studies on psychosocial interventions to prolong its efficacy. Table 1 summarizes the related hypotheses described in this editorial. Research on ketamine's effect on cognition will base such efforts on empirically driven hypotheses. 
TABLE 1. Hypotheses on specific ways to combine ketamine and psychotherapy

\begin{tabular}{|c|c|c|c|}
\hline Ketamine's neurobiological effect & Ketamine's clinical effect & $\begin{array}{l}\text { Ketamine's psychological } \\
\text { effect }\end{array}$ & $\begin{array}{l}\text { Combination with } \\
\text { psychotherapy }\end{array}$ \\
\hline $\begin{array}{l}\text { Neuroplasticity in human reward } \\
\text { pathways } \uparrow\end{array}$ & Anhedonia $\downarrow$ & Reinforcement learning $\uparrow$ & Behavioral activation \\
\hline Anti-inflammatory effects & $\begin{array}{l}\text { Social avoidance } \downarrow \text { Rejection } \\
\text { sensitivity } \downarrow\end{array}$ & Therapeutic bond $\uparrow$ & $\begin{array}{l}\text { Interpersonal } \\
\text { psychotherapy, etc. }\end{array}$ \\
\hline Lateral habenula bursting $\downarrow$ & Pessimistic thinking $\downarrow$ & $\begin{array}{l}\text { Bias toward negative } \\
\text { information } \downarrow\end{array}$ & Cognitive bias modification \\
\hline $\begin{array}{l}\text { Effects on hippocampus and prefrontal } \\
\text { cortex }\end{array}$ & Specific cognitive deficits & Disruption of fear memory & $\begin{array}{l}\text { Trauma-focused } \\
\text { psychotherapies }\end{array}$ \\
\hline $\begin{array}{l}\text { Neuroplasticity in thalamocortical } \\
\text { circuits } \uparrow\end{array}$ & Perception $\uparrow$ Memory $\uparrow$ & $\begin{array}{l}\text { Personality dimension } \\
\text { openness } \uparrow\end{array}$ & Humanistic psychotherapies \\
\hline
\end{tabular}

\section{Disclosures}

Gregor Hasler has received support from Swiss National Foundation, University of Bern, Switzerland, and University of Fribourg, Switzerland.

\section{REFERENCES:}

1. Craighead WE, Dunlop BW. Combination psychotherapy and antidepressant medication treatment for depression: for whom, when, and how. Annu Rev Psychol. 2014;65: 267-300.

2. Cuijpers P, van Straten A, Warmerdam L, et al. Psychotherapy versus the combination of psychotherapy and pharmacotherapy in the treatment of depression: a meta-analysis. Depress Anxiety. 2009;26(3): 279-288.

3. Duman RS, Aghajanian GK, Sanacora G, et al. Synaptic plasticity and depression: new insights from stress and rapid-acting antidepressants. Nat Med. 2016;22(3): 238-249.

4. Costi S, Soleimani L, Glasgow A, et al. Lithium continuation therapy following ketamine in patients with treatment resistant unipolar depression: a randomized controlled trial. Neuropsychopharmacology. 2019. Epub ahead of print.

5. Krystal JH, Abdallah CG, Sanacora G, et al. Ketamine: a paradigm shift for depression research and treatment. Neuron. 2019;101(5): 774-778.

6. Thomas RK, Baker G, Lind J,et al. Rapid effectiveness of intravenous ketamine for ultraresistant depression in a clinical setting and evidence for baseline anhedonia and bipolarity as clinical predictors of effectiveness. J Psychopharmacol. 2018;32(10): 1110-1117.
7. Ballard ED,Wills K, Lally N, et al. Anhedonia as a clinical correlate of suicidal thoughts in clinical ketamine trials. J Affect Disord. 2017;218: 195-200.

8. Lopresti AL. Cognitive behaviour therapy and inflammation: a systematic review of its relationship and the potential implications for the treatment of depression. Aust N Z J Psychiatry. 2017;51(6): 565-582.

9. Hasler G, Drevets WC, Manji HK, et al. Discovering endophenotypes for major depression. Neuropsychopharmacology. 2004;29(10): 1765-1781.

10. Yang Y, Cui Y, Sang K, et al. Ketamine blocks bursting in the lateral habenula to rapidly relieve depression. Nature. 2018;554(7692): 317-322.

11. Fattore L, Piva A, Zanda MT, et al. Psychedelics and reconsolidation of traumatic and appetitive maladaptive memories: focus on cannabinoids and ketamine. Psychopharmacology (Berl). 2018;235(2): 433-445.

12. MacLean KA, Johnson MW, Griffiths RR. Mystical experiences occasioned by the hallucinogen psilocybin lead to increases in the personality domain of openness. J Psychopharmacol. 2011;25(11): 1453-1461.

13. Rogers CR. The concept of the fully functioning person. Psychother Theory Res Pract. 1963;1(1): 17-26.

14. Stocker K, Hasler G, Hartmann M. The altered-state-ofconsciousness aspect of a feeling of lightness is reported to be associated with antidepressant benefits by depressed individuals receiving ketamine infusions: a systematic analysis of Internet video testimonials. Psychother Psychosom. 2019:1-2. Epub ahead of print.

15. Kupferberg A, Bicks L, Hasler G. Social functioning in major depressive disorder. Neurosci Biobehav Rev. 2016;69: 313-332. 